\title{
Two novel NPHS1 mutations in a Chinese family with congenital nephrotic syndrome
}

\author{
L.Q. Wu*, J.J. Hu*, J.J. Xue and D.S. Liang \\ State Key Laboratory of Medical Genetics, Xiangya Hospital, \\ Central South University, Changsha, Hunan, China \\ *These authors contributed equally to this study. \\ Corresponding author: D.S. Liang \\ E-mail: liangdesheng@sklmg.edu.cn
}

Genet. Mol. Res. 10 (4): 2517-2522 (2011)

Received December 1, 2010

Accepted August 25, 2011

Published October 18, 2011

DOI http://dx.doi.org/10.4238/2011.October.18.1

\begin{abstract}
Congenital nephrotic syndrome of the Finnish type (CNF) is a lethal, autosomal recessive disorder mainly caused by mutations in the NPHS1 gene; it is found at a relatively high frequency in Finns. We investigated the disease-causing mutations in a Chinese family with $\mathrm{CNF}$ and developed a prenatal genetic diagnosis for their latest pregnancy. Mutation analysis was made of all exons and exon/intron boundaries of NPHS1 in the fetus, parents and 50 unrelated controls using PCR and direct sequencing. A heterozygous nonsense mutation within exon 20 (c.2783C $>\mathrm{A})$ and a missense mutation within exon 17 (c.2225T $>$ C) in NPHS1 were detected in the proband's father and mother, respectively, but were not found in the fetus or in 50 unrelated controls. Two novel mutations of c.2783C $>\mathrm{A}$ and c.2225T $>\mathrm{C}$ in NPHS1 were found to be causative in this Chinese CNF family with no known Finnish ancestry. The most recent sibling did not inherit these two mutations and hence was unaffected with CNF. Determining the cumulative number and ethnic distribution of known mutations can help expedite further study of the pathogenesis of CNF.
\end{abstract}

Key words: Congenital nephrotic syndrome of Finnish type; NPHS1; Mutation analysis; Prenatal genetic diagnosis; Chinese family 


\section{INTRODUCTION}

Congenital nephrotic syndrome of the Finnish type (CNF) is a rare autosomal recessive kidney disease first and most thoroughly described in Finnish communities. Homozygous mutations in the NPHS1 gene encoding nephrin account for $-40 \%$ of infants with congenital nephrotic syndrome (CNS; Schoeb et al., 2010). Two thirds of patients with manifestations in the first year of life show mutations in four genes (NPHS1, NPHS2, WT1, or LAMB2), but NPHS1 mutations occur only in CNS (Hinkes et al., 2007).

The disease develops in utero and is diagnosed soon after birth, always presenting before three months of age on the basis of massive proteinuria and a large placenta weighing over $25 \%$ of the child's birth weight (Patrakka et al., 2000). The course of the disease is progressive, often leading to end-stage renal disease (ESRD) by two or three years of age. NPHS1 is relatively common in the Finnish population, with an incidence of 1:8200 newborns (Ahvenainen et al., 1956; Norio, 1966). There is a much lower frequency in other countries (Fuchshuber et al., 1996); and there are no CNF statistical data for China.

A nonconsanguineous Chinese couple with pregnancy, having had two children die of ESRD as well as having suffered a spontaneous abortion of a possibly affected fetus, was referred to us for genetic prenatal diagnosis. We endeavored to investigate disease-causing mutations in the family and perform prenatal genetic diagnosis and counseling for their current pregnancy. Examination protocols were approved by the Central South University Ethics Committees. Informed consent was obtained from the family members.

\section{SUBJECTS AND METHODS}

\section{Patients}

CNS had been diagnosed in two siblings of a Chinese family (Figure 1) with no known Finnish ancestry. One sibling (II-1) weighed normally at birth after an uneventful 38 -week pregnancy of the primigravid mother. The weight of the placenta was described to be heavier than usual. Consanguinity was denied and no chemical exposure or maternal drug was noted during the pregnancy. The proband developed normally until the 10th day of life when he was hospitalized with abdominal distension and myasthenia of limbs. He had normal male external genitalia. Laboratory investigation showed heavy proteinuria $(+++)$, low serum albumin and hyperlipemia. The proband died of ESRD at the age of one month.

The proband's sister (II-3) was born at 37 weeks because of premature rupture of membranes, and the weight of the placenta was heavier than usual. At the age of 20 days, she presented the same symptoms as the proband. Laboratory data are given in Table 1. Although the condition was improved by administration of albumin, she died of ESRD at the age of three months.

The parents had been clinically evaluated as normal at Xiangya Hospital of Central South University. They were experiencing their fourth pregnancy at 20 weeks gestation and were referred to us for prenatal genetic diagnosis. 
I

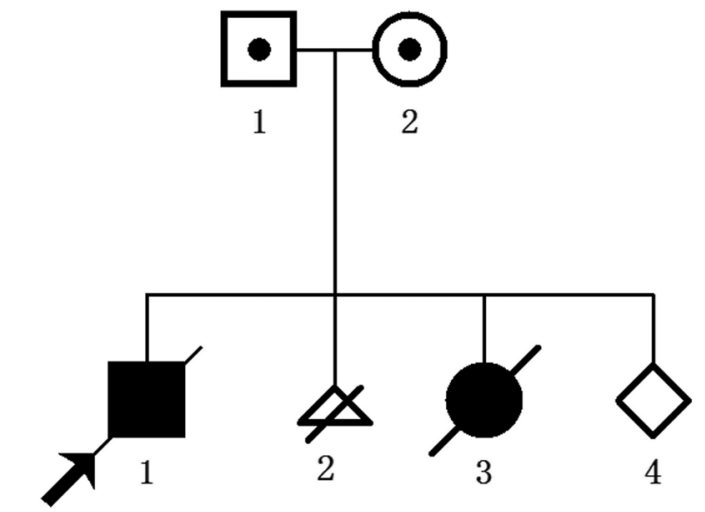

Figure 1. The Chinese congenital nephrotic syndrome pedigree.

\begin{tabular}{|c|c|c|c|c|}
\hline Inspection item & Results & Status & Reference value & Unit \\
\hline Total protein & 26.6 & $\downarrow$ & $64-83$ & $g / L$ \\
\hline Albumin & 18.2 & $\downarrow$ & $35-55$ & $g / L$ \\
\hline Globulin & 8.8 & $\downarrow$ & $20-30$ & $g / L$ \\
\hline Calcium & 1.6 & $\downarrow$ & $2.1-2.6$ & $\mathrm{mM}$ \\
\hline Chloride & 94.0 & $\downarrow$ & $98-109$ & $\mathrm{mM}$ \\
\hline Magnesium & 0.5 & $\downarrow$ & $0.7-1.15$ & $\mathrm{mM}$ \\
\hline Serum sodium & 125.0 & $\downarrow$ & $137-147$ & $\mathrm{mM}$ \\
\hline Phosphorus & 1.61 & $\uparrow$ & $0.7-1.5$ & $\mathrm{mM}$ \\
\hline Total cholesterol & 9.0 & $\uparrow$ & $3-6$ & $\mathrm{mM}$ \\
\hline Creatinine & 19.0 & $\downarrow$ & $70.7-106.1$ & $\mu \mathrm{M}$ \\
\hline Urea & 1.4 & $\downarrow$ & $8-21$ & $\mathrm{mM}$ \\
\hline Uric acid & 72.0 & $\downarrow$ & $150-430$ & $\mu \mathrm{M}$ \\
\hline Total carbon dioxide & 36.0 & $\uparrow$ & $22-31$ & $\mathrm{mM}$ \\
\hline
\end{tabular}

\section{Genetic analysis}

Genomic DNA was extracted from peripheral blood and amniotic fluid samples using phenol-chloroform, according to standard procedures. Mutation analysis of NPHS1 and NPHS2 was performed using PCR and direct sequencing. Primers were designed to cover the sequences of all exons and introns adjacent to each exon of NPHS1 and NPHS2, according to published primer sequences (Lenkkeri et al., 1999; Tsukaguchi et al., 2002). PCR products were directly sequenced with the ABI PRISM BigDye kit using the same amplification primers on an ABI 3100 DNA sequencer. Sequences were analyzed with the DNAStar software.

Fifty unrelated healthy volunteers were recruited as controls, including 29 males and 21 females, 10-60 years old, with a mean age of 35 years. The novel missense mutations of NPHS1 were investigated in the 50 healthy controls by direct sequencing.

\section{RESULTS}

No mutation of NPHS2 was detected in the parents. Two heterozygous single-base 
mutations (c.2289C $>$ T and c. $2783 \mathrm{C}>\mathrm{A}$ ) of $\mathrm{NPHS1}$ were found in the father. While not published previously, the c.2289C $>\mathrm{T}$ is a synonymous mutation less likely to cause disease. The c. $2783 \mathrm{C}>\mathrm{A}$ (Figure $2 \mathrm{~A}$ ) within exon 20 has not been previously reported; it causes a premature termination of translation, creating a truncated NPHS1 protein of 928 amino acids. This truncated nephrin has lost the Ig8, FN-III and transmembrane domains, which can seriously affect the function of nephrin protein. Thus, the c.2783C $>\mathrm{A}$ is speculated to be a nonsense and causative de novo mutation.
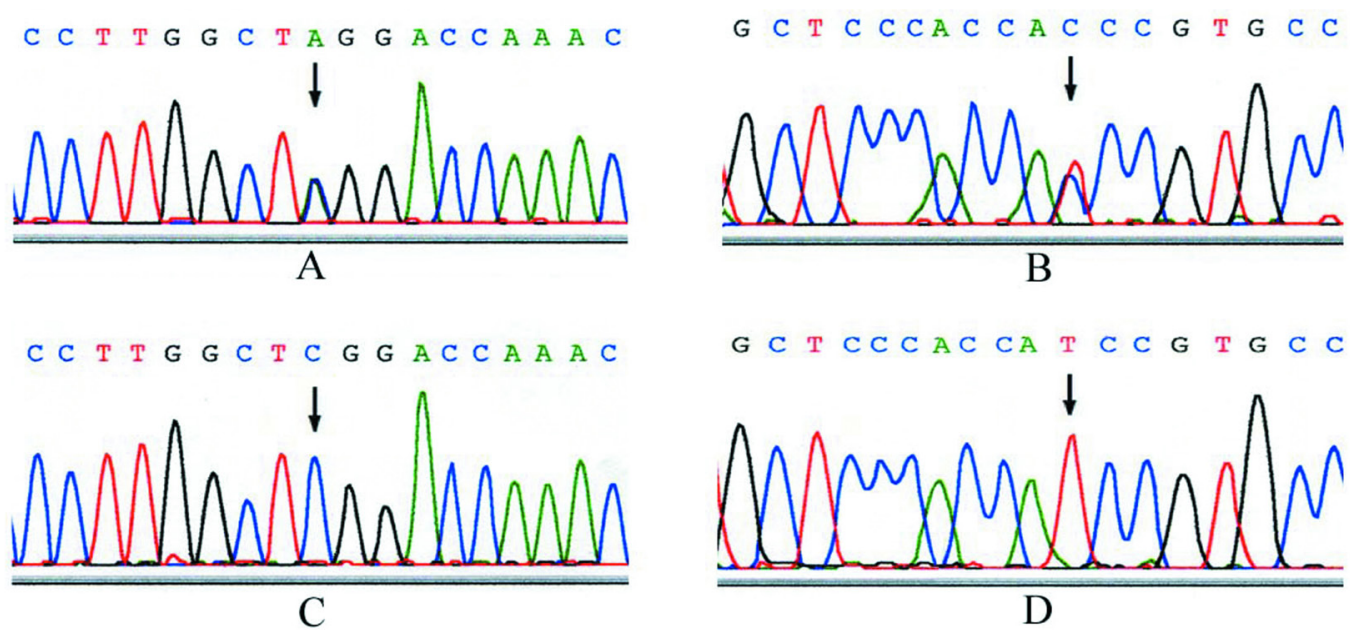

Figure 2. Sequencing of NPHS1. A. The novel nonsense mutation c. $2783 \mathrm{C}>\mathrm{A}$ (exon 20) in the father. B. The novel missense mutation c.2225T $>C$ (exon 17) in the mother. C. and D. The sequencing results for the fetus.

Two single-base mutations (c.349G $>\mathrm{A}$ and c.2225T $>\mathrm{C}$ ) of NPHS1 were detected in the mother (I-2). The c.349G $>$ A is a common SNP according to the SNP Database. The c.2225T $>$ C (Figure 2B) within exon 17 causes an amino acid substitution (p.I742T). This previously unpublished mutation was not found in the 50 controls by direct sequencing and restriction analysis. Its coded amino acid, located in $\operatorname{Ig} 7$ motif, was oriented adjacent to the reported common pathogenic mutation c.2227C $>\mathrm{T} / \mathrm{R} 743 \mathrm{C}$ (Lenkkeri et al., 1999). Moreover, the mutation site is highly conserved among humans and some other mammals. Therefore, the missense mutation c.2225T $>\mathrm{C}$ is speculated to be pathogenic, which has not been published previously.

Mutation analysis of NPHS1 was then performed for the fetus using an amniotic fluid sample for prenatal genetic diagnosis. This revealed no mutation in exons 17 or 20, indicating that the nonsense mutation c. $2783 \mathrm{C}>\mathrm{A}$ and the missense mutation c. $2225 \mathrm{~T}>\mathrm{C}$ (Figure $2 \mathrm{C}$ and D) were not inherited. Both of these mutations were absent in the 50 controls.

\section{DISCUSSION}

In 1998, mutations in NPHS1 (AF035835) were first reported to be associated with CNF by Kestilä et al. (1998). So far, more than 138 different mutations of NPHS1, involving mostly exons, have been published (Schoeb et al., 2010), of which two kinds of classical mutations were most commonly found in Finnish patients, i.e., Fin ${ }_{\text {major }}$ (nt121delCT, L41fsX91) 
in exon 2 and Fin ${ }_{\text {minor }}$ (c.3325C $>$ T, R1109X) in exon 26. Up to date, only five mutations have been described in Chinese, including one deletion (1983-1900del8) (Shi et al., 2005) and four missense mutations (D310N, Q453R, R800C, V957L) (Shi et al., 2005; Mao et al., 2007). We found two novel mutations, c. $2783 \mathrm{C}>\mathrm{A}$ and c.2225T $>\mathrm{C}$ in NPHS1, that caused CNF in a Chinese family with no known Finnish ancestry. Information concerning the frequency and ethnic distribution of known mutations will help us understand the pathogenesis of CNF.

The NPHS1 gene consists of 29 exons and has a genomic size of $26 \mathrm{~kb}$, the coding region being $4.3 \mathrm{~kb}$. The protein nephrin has 1241 amino acids and is homologous to cell adhesion molecules (CAMs) of the immunoglobulin superfamily. Several CAMs have had diverse functions in cell-cell or cell-matrix interactions attributed to them. CAMs have an extracellular domain of eight immunoglobulin-like modules, a fibronectin type III-like module, a single transmembrane domain, and a cytosolic C-terminal end (Kestilä et al., 1998). Nephrin plays a significant role in promoting cellular contacts through direct nephrin-nephrin interactions due to other slit diaphragm components' contribution (Khoshnoodi et al., 2003). Phosphorylated nephrin binds to Nck, which is an adapter protein, reorganizing the cell's actin cytoskeleton in podocytes (Jones et al., 2006).

We found that the Chinese family carried two disease-causing mutations of NPHS1, i.e., a nonsense (c. $2783 \mathrm{C}>\mathrm{A})$ and a missense $(\mathrm{c} .2225 \mathrm{~T}>\mathrm{C})$ mutation in the father and mother, respectively. The nonsense mutation $(\mathrm{c} .2783 \mathrm{C}>\mathrm{A})$ results in a truncated nephrin molecule that lacks the intracellular and transmembrane domains (Lenkkeri et al., 1999). The missense mutation (c.2225T $>$ C) in Ig7 might cause misfolding and defective intracellular transport, with consequent absence of the mutant nephrin on the plasma membrane (Liu et al., 2001). Koziell et al. (2002) reported that most mutations causing CNF with a serious clinical phenotype were in Ig2, Ig4 and Ig7 of nephrin.

Based on the clinical diagnosis of CNF in the two deceased siblings (II-1 and II-3), we presumed that they had inherited the father's nonsense mutation and mother's missense mutation concurrently. Fortunately, the results of mutation analysis showed that both parent's pathogenic mutations were not inherited by and no novel causative mutation of NPHSI was found in the current fetus (II-4), who is hence unaffected with CNF. The fetus was born and developed normally to the age of 240 days at the time we prepared this publication. Our findings indicate that, even though CNF is not very common in China, it is important to include it as a possibility in genetic counseling.

\section{ACKNOWLEDGMENTS}

We thank the participating family members for their help and support. Research supported by a grant from the National Key Technologies R\&D Program of China (\#2006BAI05A08).

\section{REFERENCES}

Ahvenainen EK, Hallman N and Hjelt L (1956). Nephrotic syndrome in newborn and young infants. Ann. Paediatr. Fenn. 2: $227-241$

Fuchshuber A, Niaudet P, Gribouval O, Jean G, et al. (1996). Congenital nephrotic syndrome of the Finnish type: linkage to the locus in a non-Finnish population. Pediatr. Nephrol. 10: 135-138.

Hinkes BG, Mucha B, Vlangos CN, Gbadegesin R, et al. (2007). Nephrotic syndrome in the first year of life: two thirds of 
cases are caused by mutations in 4 genes (NPHS1, NPHS2, WT1, and LAMB2). Pediatrics 119: e907-e919.

Jones N, Blasutig IM, Eremina V, Ruston JM, et al. (2006). Nck adaptor proteins link nephrin to the actin cytoskeleton of kidney podocytes. Nature 440: 818-823.

Kestilä M, Lenkkeri U, Männikkö M, Lamerdin J, et al. (1998). Positionally cloned gene for a novel glomerular protein nephrin - is mutated in congenital nephrotic syndrome. Mol. Cell 1: 575-582.

Khoshnoodi J, Sigmundsson K, Ofverstedt LG, Skoglund U, et al. (2003). Nephrin promotes cell-cell adhesion through homophilic interactions. Am. J. Pathol. 163: 2337-2346.

Koziell A, Grech V, Hussain S, Lee G, et al. (2002). Genotype/phenotype correlations of NPHS1 and NPHS2 mutations in nephrotic syndrome advocate a functional inter-relationship in glomerular filtration. Hum. Mol. Genet. 11: 379-388.

Lenkkeri U, Männikkö M, McCready P, Lamerdin J, et al. (1999). Structure of the gene for congenital nephrotic syndrome of the finnish type (NPHS1) and characterization of mutations. Am. J. Hum. Genet. 64: 51-61.

Liu L, Doné SC, Khoshnoodi J, Bertorello A, et al. (2001). Defective nephrin trafficking caused by missense mutations in the NPHS1 gene: insight into the mechanisms of congenital nephrotic syndrome. Hum. Mol. Genet. 10: 2637-2644.

Mao J, Zhang Y, Du L, Dai Y, et al. (2007). NPHS1 and NPHS2 gene mutations in Chinese children with sporadic nephrotic syndrome. Pediatr. Res. 61: 117-122.

Norio R (1966). Heredity in the congenital nephrotic syndrome. A genetic study of 57 Finnish families with a review of reported cases. Ann. Paediatr. Fenn. 12: 1-94.

Patrakka J, Kestila M, Wartiovaara J, Ruotsalainen V, et al. (2000). Congenital nephrotic syndrome (NPHS1): features resulting from different mutations in Finnish patients. Kidney Int. 58: 972-980.

Schoeb DS, Chernin G, Heeringa SF, Matejas V, et al. (2010). Nineteen novel NPHS1 mutations in a worldwide cohort of patients with congenital nephrotic syndrome (CNS). Nephrol. Dial. Transplant. 25: 2970-2976.

Shi Y, Ding J, Liu JC, Wang H, et al. (2005). NPHS1 mutations in a Chinese family with congenital nephrotic syndrome. Zhonghua Er Ke Za Zhi 43: 805-809.

Tsukaguchi H, Sudhakar A, Le TC, Nguyen T, et al. (2002). NPHS2 mutations in late-onset focal segmental glomerulosclerosis: R229Q is a common disease-associated allele. J. Clin. Invest. 110: 1659-1666. 